\title{
Determination of nitrofurans in animal feeds by liquid chromatography-UV photodiode array detection and liquid chromatography-ionspray tandem mass spectrometry
}

\author{
Jorge Barbosa ${ }^{a}$, Sara Moura ${ }^{\mathrm{a}}$, Rita Barbosa ${ }^{\mathrm{a}}$, Fernando Ramos ${ }^{\mathrm{b}, *}$, \\ Maria Irene Noronha da Silveira ${ }^{b}$ \\ ${ }^{a}$ LNIV, Laboratório Nacional de Investigação Veterinária, Estrada de Benfica, 701, 1549-011 Lisboa, Portugal \\ ${ }^{\mathrm{b}}$ Grupo de Bromatologia, Centro de Estudos Farmacêuticos, Faculdade de Farmácia da Universidade de Coimbra, 3000-295 Coimbra, Portugal \\ Received 16 June 2006; received in revised form 25 October 2006; accepted 16 November 2006 \\ Available online 28 November 2006
}

\begin{abstract}
Within the EU, the use of nitrofurans is prohibited in food production animals. For this reason detection of these compounds in feedingstuffs, at whatever limit, constitutes an offence under EU legislation. This detection generally involves the use of analytical methods with limits of quantification lowers than $1 \mathrm{mg} \mathrm{kg}^{-1}$. These procedures are unsuitable for the detection and confirmation of trace amounts of nitrofurans in feedingstuffs due to contamination. It is well known that very low concentrations of these compounds can be the source of residues of nitrofuran metabolites in meat and other edible products obtained from animals consuming the contaminated feed. The present multi-compound method was capable of measuring very low concentrations of nitrofurantoin (NFT), nitrofurazone (NFZ), furazolidone (FZD) and furaltadone (FTD) in animal feed using nifuroxazide (NXZ) as internal standard. Following ethyl acetate extraction at mild alkaline conditions and purification on $\mathrm{NH}_{2}$ column, the nitrofurans are determined using liquid chromatography with photodiode-array detection (LC-DAD). It was observed a CC $\alpha$ ranged from 50 to $100 \mu \mathrm{g} \mathrm{kg}^{-1}$. The liquid chromatography-tandem mass spectrometric (LC-MS/MS) procedure was used to confirm the identity of the suspected presence of any of the nitrofuran compounds.
\end{abstract}

(C) 2006 Elsevier B.V. All rights reserved.

Keywords: Nitrofurans; Feeds; Liquid-chromatography; Photo-diode array detection; Tandem mass spectrometry

\section{Introduction}

Nitrofurans are Schiff's base derivatives of nitrofuraldehyde known to have a broad-spectrum of antimicrobial activity. They were widely used as feed additives in food-producing animals like poultry, swine, cultured fish and shrimps, for treatment and prevention of various gastrointestinal infections caused by bacteria or protozoa and as growth promoters [1]. A great advantage of these compounds in comparison with other antimicrobial agents, is the slowly development and only to a limited extent of the in vivo bacterial resistance. The most common nitrofurans are furazolidone (FZD), nitrofurantoin (NFT), nitrofurazone (NFZ) and furaltadone (FTD) (Fig. 1). These compounds are rapidly metabolized in vivo, leading to a significant decrease of their par-

\footnotetext{
* Corresponding author. Tel.: +351 239859994; fax: +351 239827126 E-mail address: fjramos@ci.uc.pt (F. Ramos).
}

ent compounds levels in plasma. The elimination half-lives of nitrofurans are very short, and some hours after administration it is almost impossible to detect any residues of parent compounds in edible tissues. A concomitant accumulation of their protein-bound residues is observed, and their detection is possible over large periods of time [2-4]. Based on the evidence of carcinogenic and genotoxic effects of these bound metabolites [5], European Union (EU) has forbidden, for more than a decade, the use of nitrofuran drugs in food producing animals [6].

The detection of nitrofuran metabolite residues in large amount of samples from poultry and aquaculture products imported to Europe from some Southeast Asian and South American countries, and also within the EU in pork and poultry meat, provoked the so called nitrofuran crisis during 2002-2003 [7]. This circumstance had demonstrated the necessity of an effective control of the illegal use of these compounds. The EU Commission Decision of 13 March, 2003 [8] was established the minimum required performance limit (MRPL) of $1 \mu \mathrm{g} \mathrm{kg}^{-1}$ for 
<smiles>O=C1OC(CN2CCOCC2)CN1/N=C/c1ccc([N+](=O)[O-])o1</smiles>

Furaltadone (FTD)<smiles>O=C1CN(/N=C/c2ccc([N+](=O)[O-])o2)C(=O)N1</smiles>

Nitrofurantoin (NFT)<smiles>N#[W]OC(=O)c1ccc(O)cc1</smiles><smiles>C=C(/C=N/N1CCOC1=O)OC(=C)[N+](=O)[O-]</smiles>

Furazolidone (FZD)<smiles>NC(=O)NN=Cc1ccc([N+](=O)[O-])o1</smiles>

Nitrofurazone (NFZ)

Fig. 1. Chemical structure of furaltadone, furazolidone, nitrofutantoin, nitrofurazone and nifuroxazide (IS).

each nitrofuran metabolite based on the great efficiency of liquid chromatography tandem mass spectrometry (LC-MS/MS) methodologies for detecting bound nitrofuran metabolites in edible tissues.

Nitrofuran compounds were usually administered to animals by means of medicated feeds or at drinking water. Concentrations in feeds ranging from 8 to $400 \mathrm{mg} \mathrm{kg}^{-1}$ were considered appropriate depending of the intended use. However, McCracken et al. [9] have confirmed the possibility for the detection of very low concentrations of nitrofuran metabolites in animal tissues exposed to a diet of contaminated feeds with furazolidone and furaltadone at levels as low as $30 \mu \mathrm{g} \mathrm{kg}^{-1}$.

This means that low concentrations of these compounds can be the source of nitrofuran residue metabolites in meat and other edible products obtained from animals consuming the contaminated feed. So, animal feedingstuffs must be analyzed with analytical procedures capable of measuring very low concentrations of nitrofurans to assure its "fit-for-purpose". Nevertheless, the majority procedures of liquid chromatography with ultraviolet detection (LC-UV) available for nitrofuran feed control, shown limits of quantification around $1 \mathrm{mg} \mathrm{kg}^{-1}$, were usually applied for a single nitrofuran compound and were not suitable for nitrofuran multicomponent screening and confirmation procedures [10-12]. The aim of this paper was to develop a methodology for screening and confirmation the presence of furazolidone (FZD), nitrofurantoin (NFT), furaltadone (FTD) and nitrofurazone (NFZ) at low concentrations in animal feed, in order to satisfy the above referred needs on feed control.

\section{Experimental}

\subsection{Reagents and materials}

All chemicals and solvents used were of analytical reagent grade except solvents used in mobile phase that were HPLC grade. Deionized water was obtained from a Mill-Q System (Millipore, Bedford, USA). Ethyl acetate, glacial acetic acid, ammonium hydroxide solution containing $\mathrm{NH} 3>30 \%$, acetone, methanol, acetonitrile, $N, N$-dimethylformamide and ammonium acetate were supplied by VWR (Darmstadt, Germany). HPLC solvents were filtered through a $0.45 \mu$ m nylon membrane (Whatman, Maidstone, USA) and Sep-Pack NH2 Cartridges (6 mL, $1 \mathrm{~g}$ ) were purchase from Waters (Milford, USA). PVDF Mini-uniprep ${ }^{\mathrm{TM}}$ vial were purchased from Whatman.

A Retsch ZM 200 miller (Haan, Germany), Mettler Toledo PC2000 and AE100 balances (Greifensee, Switzerland), a Heraeus Megafuge 1.0 centrifuge (Hanau, Germany), a rotary vacuum evaporator (Büchi, Flawil, Switzerland) and a speedVac concentrator (Thermo electron corporation, Milford, USA) were used to prepare samples, to perform extraction and clean-up procedures.

Liquid chromatography with diode array detection (LCDAD) determinations were performed in an HP/Agilent 1100 Series HPLC system with a diode array detector (HP/Agilent Techonologies, Waldbronn, Germany), using a Lichrospher 60, RP-select B, $5 \mu \mathrm{m}, 250 \mathrm{~mm} \times 4 \mathrm{~mm}$ analytical column with a Lichrospher 60 , RP-select B, $5 \mu \mathrm{m}, 4 \mathrm{~mm} \times 4 \mathrm{~mm}$ preguard column (Merck, Darmstadt, Germany). Data acquisition was controlled by a ChemStation for LC 3D ${ }^{\circledR}$ software, rev. A.10.01 (Agilent Techonologies, Waldbronn, Germany).LCMS/MS was performed in an Agilent 1100 Series HPLC system (Agilent Techonologies, Waldbronn, Germany) coupled to a Triple Quadrupole System Sciex API 3000 (Applied Biosystem, Foster City, USA) tandem mass detector, with a Zorbax Eclipse $\mathrm{XDB}-\mathrm{C} 18,5 \mu \mathrm{m}, 150 \mathrm{~mm} \times 2.1 \mathrm{~mm}$ column, with a guard column Zorbax Eclipse XDB, C8, $5 \mu \mathrm{m}, 12.5 \mathrm{~mm} \times 2.1 \mathrm{~mm}$ (Agilent Technology, Palo Alto, USA). Data acquisition was controlled by a Sciex Analyst ${ }^{\circledR}$ software, Version 1.4.1 (Applied Biosystems, Foster City, CA, USA).

\subsection{Standard solutions}

Furazolidone, furaltadone, nitrofurantoin, nitrofurazone and nifuroxazide, which were used as internal standard 
(IS), were all purchased from Sigma-Aldrich (St. Louis, USA).

Stock standard solutions of the five compounds $\left(100 \mu \mathrm{g} \mathrm{mL}^{-1}\right)$ were prepared by dissolving each one in $\mathrm{N}, \mathrm{N}$-dimethylformamide:methanol (10:90). Working mixed standard solutions of the compounds were prepared by diluting the stock standard solutions in mobile phase $(14 \mathrm{mM}$ ammonium acetate $\mathrm{pH}$ 4.6:acetonitrile (70:30, v:v)). Work standard solutions were stable at least for 2 months when stored in the dark at $4{ }^{\circ} \mathrm{C}$. Stock solutions were stored in the dark at $-20^{\circ} \mathrm{C}$ and were stable at least for 6 months.

\subsection{Samples}

Poultry, porcine and bovine feed samples were commercial feeds available on the Portuguese market.

\subsection{Extraction}

An amount of $5.0 \mathrm{~g}$ thoroughly minced feed was weighed into a $250 \mathrm{~mL}$ polypropylene copolymer centrifuge flask and spiked with IS at a concentration of $500 \mu \mathrm{g} \mathrm{kg}^{-1}$. Then, $20 \mathrm{~mL}$ of ammonium acetate $79 \mathrm{mM}$ solution $(\mathrm{pH} 4.6)$ were added and the $\mathrm{pH}$ was adjusted to 8 with ammonia hydroxide solution $\geq 30 \%$. The mixture was allowed to rest for $15 \mathrm{~min}$. Ethyl acetate $(30 \mathrm{~mL})$ was added before stirring for $20 \mathrm{~min}$ in a rotary shaker and centrifuged for $10 \mathrm{~min}$ at $3000 \mathrm{rpm}$. The organic layer was collected and evaporated to dryness in a rotary vacuum evaporator at $35^{\circ} \mathrm{C}$ and $240 \mathrm{mbar}$. The resulting extract is reconstituted in $2 \mathrm{~mL}$ of a mixture of acetone and methanol 80:20 (v:v).

\subsection{Clean-up}

A Sep-Pack NH2 cartridge was conditioned with $5 \mathrm{~mL}$ of a mixture of acetone:methanol $(80: 20, \mathrm{v}: \mathrm{v})$. The reconstituted extract was put onto the cartridge and, then, the nitrofurans were eluted with $5 \mathrm{~mL}$ of the previous mixture. The eluate was evaporated to dryness in the speedVac concentrator and the residue was reconstituted with $500 \mu \mathrm{L}$ of a mixture of ammonium acetate solution $14 \mathrm{mM}$ ( $\mathrm{pH} 4.6)$ :acetonitrile (70:30, v:v).

The resulting solution was filtered through a $0.45 \mu \mathrm{m}$ PVDF Mini-uniprep ${ }^{\mathrm{TM}}$ vial before chromatography.

\subsection{LC-DAD analysis}

$50 \mu \mathrm{L}$ of the filtrate was injected into the LC-DAD system. A binary gradient mobile phase composed by a mixture of $14 \mathrm{mM}$ ammonium acetate ( $\mathrm{pH}$ 4.6) (A) and acetonitrile (B) was used at a flow rate of $1.2 \mathrm{~mL} \mathrm{~min}^{-1}$. The gradient starts with $70 \%$ (A) for $1 \mathrm{~min}$, linearly decreased to $50 \%$ (A) in $15 \mathrm{~min}$, brought back to $70 \%$ (A) in $0.1 \mathrm{~min}$ and maintaining these conditions for $3.9 \mathrm{~min}$ with a total run time of $20 \mathrm{~min}$. Detection was made at $375 \mathrm{~nm}$ for all the compounds, because all the analyzed nitrofurans show a maximum absorbance near this wavelength.
Table 1

Precursor/product ions monitored in the LC-MS/MS

\begin{tabular}{lll}
\hline Compound & Precursor ion $(\mathrm{m} / \mathrm{z})$ & Product ion $(\mathrm{m} / \mathrm{z})$ \\
\hline Positive mode & & \\
$\quad$ Furaltadone (FTD) & 325.00 & $251.92, \underline{280.99}$ \\
Furazolidone (FZD) & 226.20 & $\underline{\underline{121.99}}, \underline{139.16}$ \\
$\quad$ Nifuroxazide (NXZ) (IS) & 276.28 & $\underline{120.98}$ \\
Negative mode & & $123.60, \underline{151.97}$ \\
$\quad$ Nitrofurantoin (NFT) & 236.97 & $\underline{123.78}, 149.65$ \\
$\quad$ Nitrofurazone (NFZ) & 196.94 & $\underline{153.86}$ \\
$\quad$ Nifuroxazide (NXZ) (IS) & 274.04 & \\
\hline
\end{tabular}

The most intense ion is underlined.

\subsection{LC-MS/MS analysis}

For LC-MS/MS analysis, injection volume was $50 \mu \mathrm{L}$ and column temperature was set at $30^{\circ} \mathrm{C}$. The binary gradient phase that was used for HPLC-DAD, was also used for LC-MS/MS but at a flow rate of $0.4 \mathrm{~mL} \mathrm{~min}^{-1}$. The gradient starts with $90 \%$ (A), linearly decreased to $10 \%$ (A) in 9 min, brought back to $90 \%$ (A) in $0.2 \mathrm{~min}$ and maintaining these conditions for $3.8 \mathrm{~min}$, with a total run time of $13 \mathrm{~min}$. The mass spectrometer was operated in electrospray positive mode for FTD, FZD and NXZ (IS) and in electrospray negative mode for NFT, NFZ and NXZ (IS) using multiple reaction monitoring mode (MRM) for data acquisition (Table 1). Nitrogen was used for curtain gas, collision gas and nebulizer gas at flow rates of 7,4 and $9 \mathrm{~L} \mathrm{~min}^{-1}$, respectively in both ionization modes. The ion source block temperature was set to $350^{\circ} \mathrm{C}$, and the electrospray capillary voltage to $5.0 \mathrm{kV}$. Declustering potential, focusing potential, collision energy and cell exit potential MS parameters were shown in Table 2. Dwell times of 600 and $200 \mathrm{~ms}$ were chosen, respectively, for nitrofurans and for internal standard evaluation.

\subsection{Confirmation}

Identification of nitrofurans in samples were performed accordingly Commission Decision 2002/657/EC criteria, namely the $\pm 2.5 \%$ tolerance for relative retention time of the analyte to that of the corresponding internal standard and observation of the tolerances set by EU criteria of peak area ratios from the controlled transitions reactions [13].

\section{Results and discussion}

Characteristic LC-DAD chromatograms of a standard mixture of the four nitrofuran compounds and internal standard (A), of a blank feed sample (B) and of a spiked feed sample (C) are shown in Fig. 2.

In order to evaluate the best conditions of the mobile phase to achieve the separation of the different nitrofurans, a mixture of acetonitrile and a dilution of primary solution of $250 \mathrm{mM}$ ammonium acetate ( $\mathrm{pH} 4.8)(70: 30, \mathrm{v}: \mathrm{v})$ was tested, with an isocratic system. In these conditions a broadening peak of the internal standard (NXZ) was observed.

It was also observed that the ammonium acetate concentration in the mobile phase interfered in the LC-MS/MS analysis, 
Table 2

LC-MS/MS parameters for declustering potential (DP), focusing potential (FP), collision energy (CE) and cell exit potential (CXP)

\begin{tabular}{|c|c|c|c|c|}
\hline Compound & DP & FP & $\mathrm{CE}$ & CXP \\
\hline Furaltadone (FTD) & 56.0 & 280.0 & $\underline{29.0}, 23.0$ & $\underline{8.0}, 8.0$ \\
\hline Furazolidone (FZD) & 61.0 & 310.0 & $\overline{21.0}, 17.0$ & $\overline{18.0}, 22.0$ \\
\hline \multicolumn{5}{|l|}{ Negative parameters } \\
\hline Nitrofurantoin (NFT) & -26.0 & -150.0 & $-20.0,-\underline{12.0}$ & $-7.0,-\underline{9.0}$ \\
\hline
\end{tabular}

by deposition of salt in the curtain plate, so a decrease in the molarity of the ammonium acetate primary solution to $14 \mathrm{mM}$ was preferred, as well as the use of a gradient separation. The $\mathrm{pH}$ control of this solution proved to be fundamental in order to attain a proper separation of the analytes. The $\mathrm{pH}$ range from 5.0 to 4.5 was tested, and it was concluded that $\mathrm{pH} 4.6$ attained the best separation with sharp and symmetrical peaks (Fig. 3).

For the liquid-liquid extraction of the feed at different $\mathrm{pH}$ values with ethyl acetate, $\mathrm{pH} 8$ was the most effective, both in terms of analytes recovery and at attaining cleaner extracts.

In the clean-up step, the use of $\mathrm{NH} 2$ columns and elution of the analytes with a solution of methanol and sodium phosphate buffer at $0.1 \mathrm{M} \mathrm{pH} 8.0(80: 20, \mathrm{v}: \mathrm{v})$, enabled the best recovery levels and the removal of most interfering agents. However, this step turned out to be critical due to the highly variable nature of results, as it seems, from the sample composition. Therefore, a mixture of acetone and methanol as elution solvent was pre-
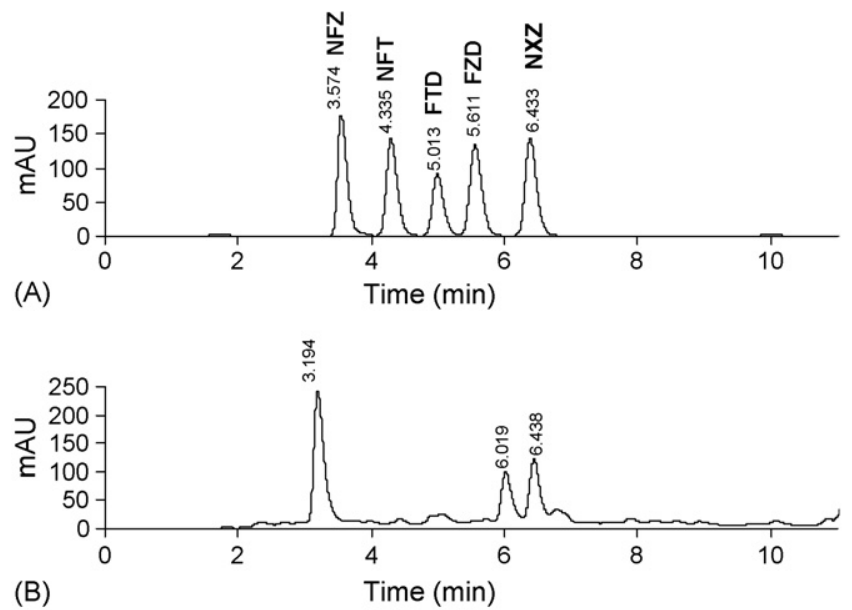

(B)

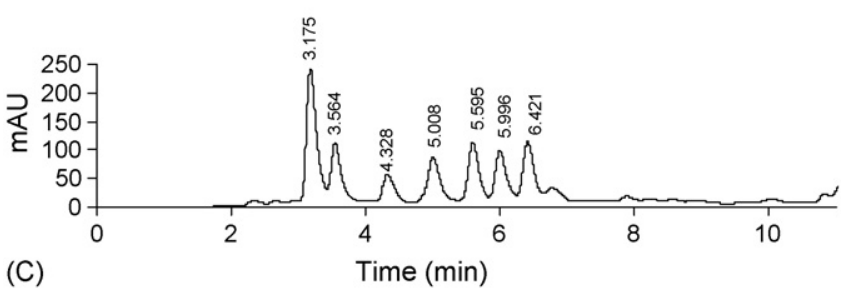

Fig. 2. LC-DAD chromatograms of (A) a mixture of nitrofuran standards at $0.5 \mu \mathrm{g} \mathrm{mL}^{-1}$; (B) blank feed; (C) blank feed fortification at $500 \mu \mathrm{g} \mathrm{kg}^{-1}$ with the four nitrofurans. ferred, even though it provided worse recoveries and less clean extracts, but with higher levels of precision.

The use of nifuroxazide as IS beside its quantitative and identification purpose, proved to be highly convenient due to the chromatographic signal attained by LC-DAD, at the monitored wavelength, and by LC-MS/MS. After attempting to optimize the conditions for NFT and NFZ by positive electrospray ionization, and as results were far from the expected, optimizing the conditions in negative electrospray ionization turned out to be the solution. It provides good results in both negative and positive ionization modes, respectively for NFT and NFZ and for FZD and FTD as it was shown in Fig. 4.

For validation, decision limit $(\mathrm{CC} \alpha)$, detection capability $(\mathrm{CC} \beta)$, specificity, repeatability, reproducibility and accuracy were determined according to Commission Decision 2002/657/EC [13].

The specificity of the method was checked by analyzing 20 different blank feed samples. These samples were randomly chosen from previously analyzed nitrofuran free samples (six from porcine, six from bovine and eight from poultry). On the expected retention times for the analytes no interfering peaks could be detected by LC-DAD and LC-MS/MS in all the analyzed samples. From these samples it was also evaluated the noise amplitude related to the internal standard signal amplitude.

A sufficient portion of each sample used to evaluate specificity was well homogenized to constituted a representative
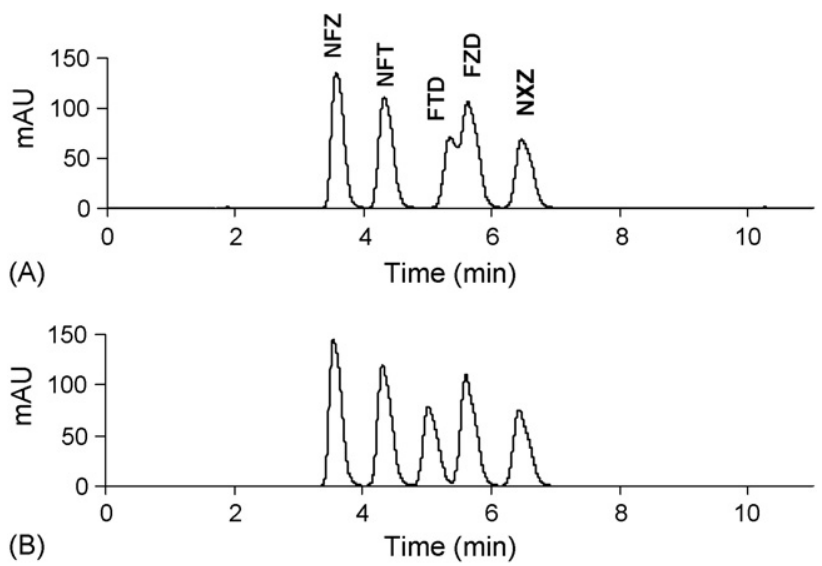

Fig. 3. LC-DAD chromatograms of a mixture of nitrofurans standards at $0.5 \mu \mathrm{g} \mathrm{mL}^{-1}$ with a primary acetate buffer solution at $\mathrm{pH} 4.9$ (A) and with a primary acetate buffer solution at $\mathrm{pH} 4.6$ (B). 


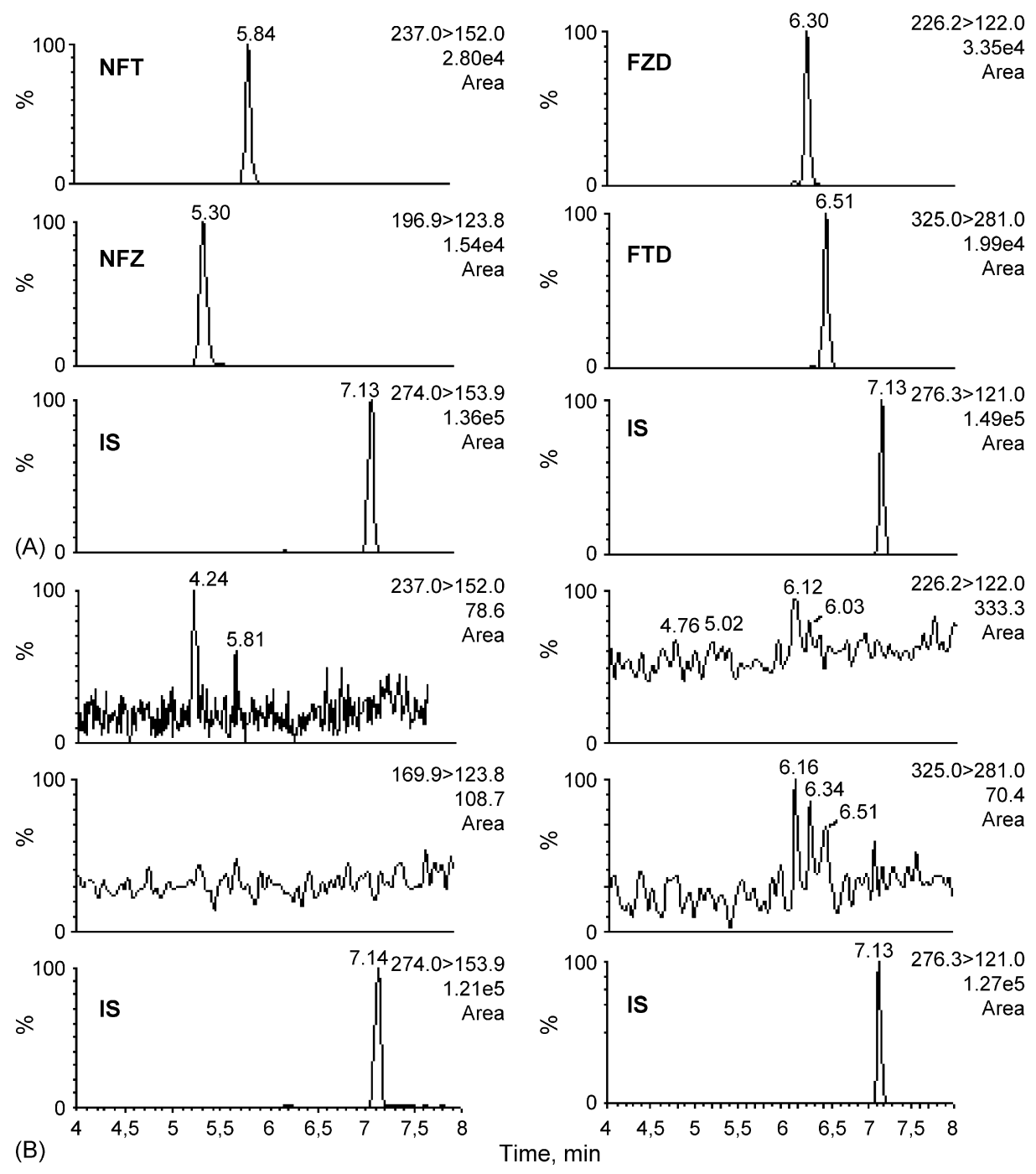

Fig. 4. (A) LC-MS/MS chromatogram of a blank feed fortified at $50 \mu \mathrm{g} \mathrm{kg}^{-1}$ with the four nitrofurans and (B) LC-MS/MS chromatogram of the blank feed.

blank sample, which was then divided into sub-samples to be used for calibration curves. The samples were prepared and analyzed on each day for 3 days. For each calibration curve, samples were fortified at different concentration levels for the four analytes. Number of spiked nitrofuran samples used in calibration curves are shown on Table 3. A standard calibration curve was also prepared at the same range of the fortified curve to be analyzed on each day [14].

Decision limit $(\mathrm{CC} \alpha)$ was calculated from results obtained from the 20 blank feed samples and from the calibration curves experiments from the 3 days, and applying the following equation (1)

$\mathrm{CC} \alpha=\frac{2.33 \sigma_{N}}{\varepsilon}$

where $\sigma_{N}$ is the standard deviation of the noise amplitude at the retention time of each analyte and $\varepsilon$ the mean slope of calibration curve [15].

For $\mathrm{CC} \beta$ calculation, the matrix-blank procedure described into Commission Decision 2002/657/EC [13] was used. For this,

Table 3

Number of spiked nitrofuran samples used in calibration curves (spiked levels are expressed in $\mu \mathrm{g} \mathrm{kg}^{-1}$ )

\begin{tabular}{|c|c|c|c|c|c|c|c|c|c|c|c|c|}
\hline \multirow[t]{2}{*}{ Spiked levels } & \multicolumn{6}{|c|}{ LC-DAD } & \multicolumn{6}{|c|}{ LC-MS/MS } \\
\hline & 0 & 100 & 150 & 200 & 300 & 500 & 0 & 10 & 20 & 30 & 50 & 100 \\
\hline Furaltadone & 1 & 6 & 6 & 6 & 1 & 1 & 1 & 6 & 6 & 6 & 1 & 1 \\
\hline Furazolidone & 1 & 6 & 6 & 6 & 1 & 1 & 1 & 6 & 6 & 6 & 1 & 1 \\
\hline Nitrofurantoin & 1 & 1 & 6 & 6 & 6 & 1 & 1 & 1 & 6 & 6 & 6 & 1 \\
\hline Nitrofurazone & 1 & 1 & 6 & 6 & 6 & 1 & 1 & 1 & 6 & 6 & 6 & 1 \\
\hline
\end{tabular}


Table 4

Validation parameters for LC-MS/MS and LC-DAD

\begin{tabular}{|c|c|c|c|c|c|c|c|c|c|c|}
\hline \multirow[t]{2}{*}{ Compound } & \multicolumn{2}{|c|}{$\mathrm{CC} \alpha\left(\mu \mathrm{g} \mathrm{kg}^{-1}\right)$} & \multicolumn{2}{|c|}{$\mathrm{CC} \beta\left(\mu \mathrm{g} \mathrm{kg}^{-1}\right)$} & \multicolumn{2}{|c|}{$\begin{array}{l}\text { Accuracy at } \\
\operatorname{CC} \beta(\%)\end{array}$} & \multicolumn{2}{|c|}{$\begin{array}{l}\text { Repeatability at } \\
\mathrm{CC} \beta(\mathrm{CV} \%)\end{array}$} & \multicolumn{2}{|c|}{$\begin{array}{l}\text { Reproducibility at } \\
\text { CC } \beta(\mathrm{CV} \%)\end{array}$} \\
\hline & MS & DAD & MS & DAD & MS & DAD & MS & DAD & MS & DAD \\
\hline Furazolidone & 10 & 51 & 30 & 150 & 68.1 & 63.1 & 8.0 & 9.1 & 11.8 & 15.1 \\
\hline Nitrofurantoin & 21 & 98 & 50 & 300 & 62.1 & 60.1 & 7.5 & 11.4 & 14.9 & 18.2 \\
\hline Nitrofurazone & 15 & 76 & 50 & 200 & 67.8 & 68.8 & 10.4 & 10.5 & 17.6 & 22.6 \\
\hline
\end{tabular}

Table 5

Calibration curve parameters in fortified feed samples at ranges of $10-100 \mu \mathrm{g} \mathrm{kg}^{-1}$ and $50-500 \mu \mathrm{g} \mathrm{kg}^{-1}$, respectively for LC-MS/MS and LCDAD

\begin{tabular}{|c|c|c|c|c|c|c|}
\hline \multirow[t]{2}{*}{ Compound } & \multicolumn{2}{|l|}{$R^{2}$} & \multicolumn{2}{|c|}{ Intercept } & \multicolumn{2}{|l|}{ Slope } \\
\hline & MS & DAD & MS & DAD & MS & DAD \\
\hline Furaltadone & 0.992 & 0.989 & 2.063 & 3.976 & 1.324 & 3.369 \\
\hline Furazolidone & 0.996 & 0.992 & 3.143 & 4.097 & 1.922 & 2.922 \\
\hline Nitrofurantoin & 0.989 & 0.985 & 2.012 & 5.972 & 1.840 & 3.638 \\
\hline Nitrofurazone & 0.990 & 0.981 & 4.865 & 4.779 & 2.701 & 3.954 \\
\hline
\end{tabular}

20 different blank feed samples where fortified at a level that were estimated during the method development to equal the $\mathrm{CC} \beta$ for the four analytes. In $95 \%$ of the samples was possible to identify the analyte. This $C C \beta$ calculation procedure was used for LC-DAD and LC-MS/MS methods. Values obtain for CC $\alpha$ and CC $\beta$ are shown in Table 4.

The linearity was evaluated by analyzing the 3 days calibration curves of fortified samples. Peak area ratios between the analytes of the interest and IS were plotted against the concentration ratios. The 3 days resulting calibration curve for each analyte show a coefficient of determination $\left(R^{2}\right)$ better than 0.98 . All calibration curve parameters are summarized in Table 5. Repeatability and within-laboratory reproducibility were evaluated at CC $\beta$ concentration. For repeatability, the coefficient of variation $(\mathrm{CV})$ of the mean concentration for the repeated analysis within the days $(n=6 \times 3)$ were calculated. For reproducibility, samples were analyzed by different operators on the 3 different days, and expressed as CV of the mean concentration between the days. Considering the application of the Horwitz equation and the criteria defined into Commission Decision 2002/657/EC [13], a CV of $23 \%$ was taken as a guide for analyte concentrations ranging from 10 to $100 \mu \mathrm{g} \mathrm{kg}^{-1}$, and all the calculated coefficient of variation were lower than the permitted CV. The accuracy for both methods was evaluated at $\mathrm{CC} \beta$ concentration by comparing data from the fortified curve and the standard calibration curve (calibration curve without matrix). The values obtained it validation data were summarized in Tables 4 and 5 .

\section{Conclusions}

The LC-UV photodiode array detection (LC-DAD) methodology that was described in this paper was appropriate for simultaneous detection of FZD, NFT, FTD and NFZ in feed samples at levels down to $50-100 \mu \mathrm{g} \mathrm{kg}^{-1}$ depending on the compound. Nonetheless, to have a positive identification by comparing the UV-spectra of the standards with those of the detected analytes, higher concentrations should be present in the sample (300-500 $\mu \mathrm{g} \mathrm{kg}^{-1}$ ) depending on the compound. When LC-MS/MS methodology described in this paper was used, detection capability is somewhat improved to levels ranging to $20-50 \mu \mathrm{g} \mathrm{kg}^{-1}$ depending on the compound. These levels were appropriate for detection of low-level contamination feed samples. Nifuroxazide (NXZ) was used as internal standard (IS) for both LC-DAD and LC-MS/MS. In routine analysis, the LCDAD method is used to screen samples for the possible presence of the four nitrofurans analyzed and the LC-MS/MS method is used to confirm any positive finding.

Finally, and based on the obtained values for precision, $\mathrm{CC} \alpha$ and CC $\beta$ in both LC-DAD and LC-MS/MS methodologies, it has been demonstrated their applicability to measure the presence of the four referred nitrofurans. Therefore, applying both described methodologies in routine analysis was simple and quick, taking into account that the same sample extract could be evaluated by LC-DAD and LC-MS/MS, for screening and confirmation for the presence of one or more of the analyzed nitrofurans.

\section{References}

[1] P. Mottier, S.-P. Khong, E. Gremaug, J. Richoz, T. Delatour, T. Goldmann, P.A. Guy, J. Chromatogr. A 1067 (2005) 85.

[2] L.H.M. Vroomen, M.J.C. Berghmans, P. van Leeuwen, T.D.B. van der Struijs, P.H.U. Vries, H.A. Kuiper, Food Addit. Contamin. 3 (1986) 331.

[3] T. Zuidema, J.A. van Rhijn, P.P.J. Mulder, Y.J.C. Bolck, L.A.P. Hoogenboom, D.G. Kennedy, Proceedings of the Euroresidue V conference, Noordewijkerhout, The Netherlands, 2004, p. 996.

[4] K.M. Cooper, P.P.J. Mulder, J.A. van Rhijn, L. Kovacsics, R.J. McCracken, P.B. Young, D.G. Kennedy, Food Addit. Contamin. 22 (2005) 406.

[5] T. Delatour, E. Gremaud, P. Mottier, J. Richoz, F.A. Vera, R.H. Stadler, J. Agric. Food Chem. 51 (2003) 6371.

[6] Commission Regulation no. 2377/90/EC, Off. J. Eur. Commun., L 224 (1990) 1.

[7] I. Diblikova, K.M. Cooper, D.G. Kennedy, M. Franek, Anal. Chim. Acta 540 (2005) 285.

[8] Commission Decision 2003/181/EC, Off. J. Eur. Commun., L71 (2003) 17.

[9] R.J. McCracken, J.A. van Rhijn, D.G. S Kennedy, Proceedings of the Euroresidue V Conference Noordewijkerhout, The Netherlands, 2004, p. 655

[10] R. Draisci, L. Giannetti, L. Lucentini, L. Palleschi, G. Brambilla, L. Serpe, P. Gallo, J. Chromatogr. A 777 (1997) 201.

[11] R.J. McCracken, D.G. Kennedy, J. Chromatogr. A 771 (1997) 349. 
[12] ISO 14797:1999, Animal feedingstuffs-Determination of furazolidone content.

[13] Commission Decision 2002/657/EC, Off. J. Eur. Commun., L221 (2002) 8.
[14] P.R. Kootstra, C.J.P.F. Kuijpers, K.L. Wubs, D. van Doorn, S.S. Sterk, L.A. van Ginkel, R.W. Stephany, Anal. Chim. Acta 529 (2005) 75.

[15] J.-P. Antignac, B. le Bizec, F. Monteau, F. André, Anal. Chim. Acta 483 (2003) 325 